\title{
Corrigendum
}

\section{Human immunodeficiency virus (HIV)-1 proteins and cytoskeleton: partners in viral life and host cell death}

\author{
P Matarrese and W Malorni \\ Cell Death and Differentiation (2006) 13, 876. \\ doi:10.1038/sj.cdd.4401893
}

Correction to: Cell Death and Differentiation (2005) 12, 932941. doi: $10.1038 /$ sj.cdd.4401582

The authors wish to cite reference 3 in an additional four places in the following text.

In addition, the dependence on intact cytoskeleton contraction also has implications for host cell selection, beyond selective coreceptor usage. ${ }^{3}$ Faced with a multitude of inappropriate potential host cells, the ability to enter only cells that are viable and activated would be a major selective advantage. $^{3}$ The idea is that cytoskeletal contraction leading to coreceptor clustering as well as an intact cell membrane could serve as an indicator of host cell fitness, which the virus probes interactively prior to entry. ${ }^{3}$ In fact, statins that alter microfilament system dynamic downregulating Rho activation also inhibit HIV-1 envelope fusion with target cell membranes, thus reducing cell infection. ${ }^{7}$ In addition, HIV mutants that are independent of cytoskeletal activation will not be pathogenic or replication competent in vivo unless they are also capable of activating quiescent cells upon binding. ${ }^{3,8}$

The authors apologise for this oversight. 